\title{
A NEW REFINEMENT OF YOUNG'S INEQUALITY
}

\author{
ABdolhossein HoORFAR AND FENG QI
}

Abstract. In this short note, the well-known Young's inequality is refined by a double inequality.

Mathematics subject classification (2000): 26D15.

Key words and phrases: Young's inequality, refinement, integral.

\section{REFERENCES}

[1] D. R. ANDERSON, Young's integral inequality on time scales revisited, J. Inequal. Pure Appl. Math. 8 (2007), no. 3, Art. 64; Available online at http://jipam.vu.edu.au/article.php?sid=876.

[2] J.-CH. KuANG, Chángyòng Bùděngshì (Applied Inequalities), 2nd ed., Hunan Education Press, Changsha, China, May 1993. (Chinese)

[3] D. S. MitRINOVIC, Analytic Inequalities, Springer-Verlag, 1970.

[4] D. S. Mitrinović, J. E. PeČARIĆ AND A. M. FinK, Classical and New Inequalities in Analysis, Kluwer Academic Publishers, 1993.

[5] Olympiad Math. Madness, Analysis, Available online at http://www.geocities.com/Cape Canaveral/Lab/4661/Frame_Calculus.html.

[6] D. Ruthing, On Young's inequality, Internat. J. Math. Ed. Sci. Techn. 25 (1994), no. 2, 161-164.

[7] T. TAKAHASHI, Remarks on some inequalities, Tôhoku Math. J. 36 (1932), 99-106.

[8] A. WitKowsKI, On Young inequality, J. Inequal. Pure Appl. Math. 7 (2006), no. 5, Art. 164; Available online at http://jipam.vu.edu.au/article.php?sid=782.

[9] W. H. Young, On classes of summable functions and their Fourier series, Proc. Roy. Soc. London Ser. A 87 (1912), 225-229.

[10] L. ZHU, On Young's inequality, Internat. J. Math. Ed. Sci. Tech. 35 (2004), no. 4, 601-603. 